\title{
MODELO DE ENFERMAGEM BASEADO NAS ATIVIDADES DE VIDA DIÁRIA: ADOLESCENTE DIABÉTICA E DEFICIENTE VISUAL ${ }^{a}$ Nursing model based on activities of daily living: a diabetic and visually-impaired adolescent Modelo de enfermería fundamentado en las actividades de la vida
cotidiana: adolescente con diabetes y deficiencia visual
}

\section{RESUMO}

0 diabetes mellitus, doença crônico-degenerativa, pode comprometer a retina. Objetivou-se analisar a eficácia do cuidado de enfermagem fundamentado no Modelo de Enfermagem Baseado nas Atividades da Vida Diária a uma adolescente com deficiência visual decorrente do diabetes mellitus tipo I, utilizando o estudo de caso. Os dados foram coletados em setembro 2006 por entrevistas no domicílio. Os resultados mostram ambiente doméstico inseguro devido a iluminação inadequada, nutrição alterada e déficit de conhecimento relacionado ao controle glicêmico, pressão arterial, sedentarismo e sexualidade. 0 Modelo foi válido, pois permitiu e promoveu o cuidado e comunicação objetiva entre pesquisadoras e a adolescente que apresentou boa apreensão, mostrou-se segura e autoconfiante, repercutindo em aumento da autoestima e desempenho de atividades de forma mais independente.

Palavras-chave: Diabetes Mellitus. Adolescente. Portadores de Deficiência Visual. Enfermagem.

\begin{abstract}
Diabetes mellitus, a chronic degenerative disease, can compromise the retina. This study aimed to apply the Nursing Model Based on Activities of Daily Living, involving a visually impaired adolescent girl due to type I diabetes mellitus, using a case study. Data were collected in September 2006 through home interviews. The results show an insecure home environment due to inadequate illumination, altered nutrition and knowledge deficit related to glucose control, arterial pressure, sedentariness and sexuality. The Model was a valid instrument, as it permitted and promoted care and objective communication between the researchers and the adolescent, who presented good understanding, revealed to be secure and self-confident, and leading to increased self-esteem and more independent activity performance.
\end{abstract}

Keywords: Diabetes Mellitus. Adolescent. Visually Impaired Persons. Nursing.

\section{Resumen}

La diabetes mellitus es una enfermedad crónica y degenerativa que puede ocasionar compromiso ocular afectando principalmente la retina. El objetivo del presente estudio fue analizar la eficacia del cuidado de enfermería fundamentada en un modelo de enfermería basado en las actividades de la vida cotidiana de una adolescente con deficiencia visual provocada por la diabetes mellitus tipo 1. Para tales efectos, se utilizó el método de estudio de caso. Los datos fueron obtenidos en septiembre del 2006 a través de entrevistas realizadas en el domicilio de la joven. Los resultados mostraron que existía un ambiente inapropiado en el hogar de la adolescente, causado principalmente, por una iluminación inadecuada, por la mala nutrición de la joven y por la falta de conocimiento sobre el control glicémico, la presión arterial, el sedentarismo y la sexualidad. El modelo probó ser eficiente ya que permitió y promovió el cuidado y la comunicación objetiva entre las investigadoras y la adolescente. La joven presentó un buen nivel de asimilación, se sintió segura y con auto confianza, lo que aumentó su autoestima y la ayudó a mejorar el desempeño de sus actividades diarias de una forma más independiente.

Palabras clave: Diabetes Mellitas. Adolescente. Personas con Daño Visual. Enfermería.

'Enfermeira. Mestre em Enfermagem pelo Programa de Pós-Graduação em Enfermagem da Universidade Federal do Ceará- Brasil. E-mail: camillaenfermagem@hotmail.com, ${ }^{2}$ Enfermeira. Doutora em Enfermagem. Professora Titular do Departamento de Enfermagem da UFC. Pesquisadora do CNPq- Brasil. Email: pagliuca@ufc.br, ${ }^{3}$ Enfermeira. Doutora em Enfermagem. Professora Adjunta do Departamento de Enfermagem da UFC- Brasil. E-mail: marligalvao@gmail.com 


\section{INTRODUCÃO}

A adolescência pode ser conceituada sob diferentes perspectivas: biológica, psicológica, jurídica e sociocultural. Entretanto, nenhuma destas perspectivas, isoladamente, é capaz de definir esta etapa do desenvolvimento humano.

Com base em uma perspectiva biológica, a adolescência é associada à puberdade, fase caracterizada por modificações corporais decorrentes da ação hormonal do eixo neurohipofisário. A puberdade constitui uma parte da adolescência identificada, principalmente, pela aceleração e desaceleração do crescimento físico, mudança da composição corporal, eclosão hormonal, evolução da maturação sexual. ${ }^{1}$

Quanto à faixa etária específica deste período, diferenciase conforme a instância que a define. Por exemplo, o Estatuto da Criança e do Adolescente, Lei n. ${ }^{\circ}$.069/90, circunscreve a adolescência como o período de vida dos 12 aos 18 anos de idade. De acordo com a Organização Mundial de Saúde (OMS), a adolescência é delimitada como a segunda década de vida (10 aos 19 anos) e a juventude como o período dos 15 aos 24 anos. Ao tomar por base a definição da OMS, o Ministério da Saúde define o público beneficiário como o contingente da populaçãa entre 10 e 24 anos de idade. ${ }^{2}$ Entretanto, a definição dada para caracterizar a adolescência, por uma instância da área da saúde, parte de critérios diferentes daqueles considerados por uma instância jurídica. Como os propósitos deste estudo estão afinados com a área da saúde, será tomada como referência de adolescência a definição do Ministério da Saúde, ou seja, contingente populacional dos 10 aos 24 anos.

No dia-a-dia, as ações de enfermagem podem ser direcionadas a toda população. Mas entre as ações de enfermagem desenvolvidas e voltadas para os adolescentes na Atenção Básica de Saúde sobressaem as seguintes: acompanhamento do crescimento e desenvolvimento; educação em métodos contraceptivos; pré-natal e acompanhamento emocional à adolescente gestante; prevenção de doenças sexualmente transmissíveis; imunização; prevenção ao uso indevido de drogas; aspectos educativos relacionados à promoção em saúde. Como observado, os principais assuntos abordados nas consultas de enfermagem ao adolescente referem-se aos processos sociais e riscos, comportamentos $\mathrm{e}$ valores, autoimagem e aceitação corporal, processos psicoemocionais, capacidade de negociação, anticoncepção, padrão alimentar, história familiar de doenças, atividades físicas e habilidades, alterações sistêmicas, acuidade visual e auditiva, calendário vacinal, entre outras. Embora o adolescente nem sempre saiba, ele tem direito a este acompanhamento do seu estado de saúde. ${ }^{3}$

Nesta perspectiva, é essencial a compreensão da fase da adolescência por parte das enfermeiras. A percepção das dificuldades vividas durante esta etapa da vida (mudança de relacionamento com os pais, pressões da escola, preocupações sobre o futuro emprego) e a certeza de ser a adolescência um período de transição, com a flutuação entre o desejo da independência adulta e a regressão à dependência da infância, são certamente essenciais na atuação das enfermeiras envolvidas com adolescentes sob seus cuidados. Tal como a infância, a adolescência é um estágio da vida no qual a saúde domina. Portanto, a doença parece uma realidade distante. Quando acometidos por doença, ou até incapacidade, os adolescentes vivenciam uma situação muito delicada e de difícil aceitação. Por exemplo, um adolescente que se torna deficiente após um acidente ou se descobre com diabetes mellitus ${ }^{4}$ enfrentará um doloroso processo dominado pelos mais variados sentimentos.

No caso do diabetes, é ainda mais comprometedor. 0 diabetes pode comprometer o aparelho visual, mais especificamente a retina, área do olho que recebe imagens e envia informações ao cérebro. A retina é uma estrutura localizada na parte posterior do olho, bastante vascularizada. Pessoas com diabetes têm propensão a complicações oculares, especificamente retinopatia e catarata, entre outros problemas.

$\mathrm{Na}$ retinopatia existem três estágios principais: não proliferativa, pré-proliferativa e proliferativa. Na retinopatia não proliferativa, os pacientes têm alguns prejuízos visuais, mas são poucos os riscos de desenvolver cegueira no futuro. Quanto à retinopatia pré-proliferativa, é considerada precursora da retinopatia proliferativa mais grave. Em torno de $10 \%$ a $50 \%$ dos pacientes irão desenvolver esta forma mais severa a curto prazo. Este tipo de retinopatia é o mais ameaçador à visão e é 0 estágio no qual ocorre a perda visual. Tal perda é causada por hemorragia no humor vítreo (estrutura gelatinosa responsável pelo formato do olho) ou descolamento de retina. ${ }^{5}$

Por muito tempo, o diabetes mellitus foi considerado uma doença de significado secundário para a saúde mundial. Atualmente, porém, é visto como uma das principais ameaças para a saúde humana. Determinados fatores contribuem para isto. Entre estes, mudanças no estilo de vida no último século têm resultado em um aumento dramático na incidência mundial do diabetes. Juntamente com a suscetibilidade genética, 0 aumento da doença estaria hoje mais fortemente impulsionado por conta de fatores ambientais e comportamentais, que incluem o sedentarismo, a nutrição demasiadamente rica em carboidratos e gorduras e a obesidade. ${ }^{6}$

$\mathrm{Na}$ sua formação profissional, o enfermeiro dispõe de diferentes modos de cuidar em enfermagem, tais como o Modelo de Enfermagem Baseado nas Atividades da Vida Diária. Este Modelo aponta como função específica da enfermeira ajudar o doente a evitar, aliviar, resolver ou até mesmo viver com os problemas (reais ou potencias), relacionando-os com as atividades de vida. Reconhecer que os problemas dos doentes podem ser reais ou potenciais é essencial para a enfermeira, pois, desse modo, ela não apenas responde aos problemas existentes, mas também se preocupa em evitá-los quando possivel. $^{4}$ 
Dentro deste contexto, desenvolveu-se o presente estudo com uma adolescente deficiente visual e com diabetes mellitus tipo I. Como observado, o comprometimento visual decorreu da doença base, causando a retinopatia diabética. Diante da situação, adotou-se como referencial teórico o Modelo de Enfermagem Baseado nas Atividades da Vida Diária, ${ }^{4}$ descrito a seguir.

\section{MODELO DE ENFERMAGEM BASEADO NAS ATIVIDADES DA VIDA DIÁRIA}

No Modelo de Enfermagem Baseado nas Atividades da Vida Diária existem cinco componentes essenciais: atividades de vida, duração de vida, grau de dependência/independência, fatores que influenciam as atividades de vida e individualidade no viver. Compreende uma série de atividades que, independente da idade e circunstâncias em que o indivíduo se encontra, fazem parte do complexo processo do "viver". As atividades de vida, em número de 12 atividades, constituem o conceito principal desse Modelo e são assim nomeadas: manutenção de um ambiente seguro, comunicação, respiração, alimentação, eliminação, higiene pessoal e vestuário, controle da temperatura do corpo, mobilidade, trabalho e lazer, expressão da sexualidade, sono e morte. ${ }^{4}$

Apesar desta classificação, as atividades de vida têm uma estreita relação entre si; desse modo, qualquer alteração em uma das atividades trará modificações no desempenho das demais. Cada pessoa tem uma duração de vida que vai do nascimento até a morte, e as fases de vida - lactência, infância, adolescência, idade adulta, velhice - influenciam 0 comportamento individual em cada atividade de vida. Ou seja, o modo como são desempenhadas as atividades de vida sofre enorme influência da idade em que se encontra o indivíduo. Essa compreensão é fundamental para que o cuidado seja adequado e eficaz. ${ }^{4}$

Do Modelo de Enfermagem Baseado nas Atividades da Vida Diária faz parte, ainda, o componente denominado grau de dependência/independência, o qual está intimamente relacionado com as etapas da vida e as atividades de vida. Inclui o reconhecimento da existência de estágios das etapas de vida em que uma pessoa não pode ainda (ou por várias razões não pode mais) executar certas atividades de vida de forma independente. Cada pessoa deve ter um estado de dependência/independência para cada uma das atividades de vida. No Modelo, os termos "dependência total" e "independência total" são utilizados para descrever os pólos de continuidade, e as setas indicam o movimento ocorrido em cada direção, de acordo com as circunstâncias. 0 grau de dependência/independência de um indivíduo em relação às atividades de vida não está ligado apenas às etapas da vida; ele está também intimamente associado aos os fatores que influenciam as atividades de vida. ${ }^{4}$

Embora cada pessoa desempenhe suas atividades de vida (a qualquer momento do estágio de vida e com graus vários de independência), cada uma o faz de forma diferente. Estas diferenças surgem dos fatores que influenciam as atividades de vida, descritos em cinco grupos principais: físicos, psicológicos, socioculturais, ambientais e político-econômicos. As atividades de vida, as etapas da vida, o grau de dependência/independência e os fatores que influenciam as atividades de vida estão interligados, e os cinco fatores em si próprios também. Como se pode perceber, o Modelo possui um conceito simples do processo complexo de "viver". Neste Modelo, a preocupação é a vida tal como vivida por cada indivíduo, e seu componente final - a individualidade no viver - serve para enfatizar este ponto. Quanto à individualidade, pode manifestar-se de várias maneiras, por exemplo: como; quantas vezes, onde; quando; por que éque essa pessoa desempenha determinada atividade de vida de uma forma particular; o que é que a pessoa sabe, acredita, e qual é a atitude da pessoa em relação à atividade de vida. Neste artigo, trabalhou-se de forma mais detalhada o principal componente do Modelo, qual seja, as atividades de vida. Contudo, os outros quatro componentes também foram considerados na elaboração do trabalho.

Teve-se como propósito analisar a eficácia do cuidado de enfermagem fundamentado no Modelo de Enfermagem Baseado nas Atividades da Vida Diária prestado a uma adolescente com deficiência visual decorrente da diabetes mellitus tipo I, diagnosticado há um ano.

\section{MÉTODO}

Para esta investigação utilizou-se o estudo de caso, pois tal metodologia permite a descrição aprofundada das dimensões e processo essenciais de um fenômeno contemporâneo. Desse modo, funciona como método eficaz no planejamento do cuidado em enfermagem.?

Na coleta de dados usou-se um formulário fundamentado no Modelo mediante a consulta de enfermagem no domicilio da cliente. Ocorreram quatro visitas domiciliares nos meses de outubro e novembro de 2006. A apresentação dos dados foi feita de acordo com a operacionalização do processo de enfermagem baseado no Modelo. Foram seguidas as fases de exame, planejamento, implementação do plano de enfermagem e avaliação. ${ }^{4}$ Os dados relacionados às fases de planejamento, implementação e avaliação foram expostos em um quadro e logo após analisados de acordo com literatura pertinente.

A fase de exame tem por objetivo a obtenção de informações sobre a pessoa, com revisão da informação obtida e identificação dos problemas e as prioridades entre estes. ${ }^{4}$ Nesta fase foram coletadas informações sobre dados biográficos e de saúde (nome, sexo, idade, parente mais próximo, local de residência, rede social de apoio, ocupação, crença religiosa, acontecimentos significativos de vida recentes); e sobre as atividades de vida, com identificação dos problemas reais e potenciais relacionados com essas atividades e com a descoberta de rotinas anteriores, verificando 0 que 0 doente podia ou não fazer de forma independente. 
No Modelo, o diagnóstico de enfermagem é substituído pela expressão "problemas do doente com as atividades de vida". O formulário possui espaço onde cada um dos problemas identificados pelo exame pode ser listado ao longo da atividade de vida relacionada. Ao atingir este estágio, é decidida a prioridade relativa dos problemas. Tal prioridade pode ser indicada de forma a arranjar os problemas ordenadamente ou, de forma alternativa, numerar sua prioridade. A fase de planejamento tem como objetivo a solução dos problemas atuais, aliviando aqueles possíveis de solução. ${ }^{4}$ Nesta fase, foram estabelecidos objetivos em colaboração com a doente e, quando possível, também com a família. Foi preparado, ainda, o plano de enfermagem.

A fase de implementação do plano de enfermagem, destinase à anotação daquelas intervenções "não planejadas" ou aparentemente "insignificantes", que mereçam registro em uma folha separada ou documento designado como "Notas de Enfermagem do Doente". Aúltima fase tem por finalidade avaliar o seguimento/cumprimento ou não dos objetivos traçados. ${ }^{4}$

Este artigo é parte de pesquisa mais abrangente aprovada pelo Comitê de Ética em Pesquisa da Universidade Federal do Ceará (COMEPE-UFC), nº 167/06. Desta forma, seguiram-se as normas que regulamentam pesquisas com seres humanos, do Conselho Nacional de Saúde, conforme a Resolução 196/96 do Ministério da Saúde. ${ }^{8}$ Previamente à coleta informou-se à participante a garantia do anonimato, resguardando-lhe 0 direito de interromper o estudo a qualquer tempo.

\section{RESULTADOS E DISCUSSÃO}

0 primeiro contato com a adolescente ocorreu em uma associação de cegos da cidade de Fortaleza. Marcou-se um encontro com ela neste local, e, posteriormente, estabeleceuse a data da primeira visita.

$\mathrm{Na}$ primeira visita domiciliar, foram recolhidas as informações sobre a adolescente e identificados os problemas. F.L.S, 19 anos, solteira, reside com a mãe em Fortaleza, cursa o segundo ano do ensino médio e é católica. Ao exame físico, verificou-se: ausência de sujidade e de secreção purulenta na pele, ausência de nódulos e gânglios palpáveis; órbita ocular com aparência geral característica, edema macular; narinas com ausência de secreções, mucosa e septo nasais sem alterações; boca com boa abertura, ausência de paralisia ou desvios faciais, lábios simétricos, secos e com pele íntegra, coloração rósea, ausência de cáries; língua com coloração amarelada e seca; pavilhão auditivo externo com ausência de secreções, exantemas, sujidade e corpos estranhos; acuidade auditiva normal; tórax simétrico; expansibilidade pulmonar simétrica, sem ruídos adventícios; ausência da ausculta de sopros cardíacos; abdome plano, ausência de abaulamentos, indolor à palpação, sem visceromegalias; respiração abdominal superficial; pele hidratada, hipocorada, turgor normal; movimentação dos membros superiores e inferiores normais, FR: 16 rpm, T: $36^{\circ} \mathrm{C}, \mathrm{P}: 80 \mathrm{bat} / \mathrm{min}, \mathrm{PA}: 140 / 90 \mathrm{mmHg}$.

Conforme relatou a adolescente, há um ano ela descobriu que era diabética, ao procurar por serviço médico em virtude de alterações em sua visão. Referia sintomas como: manchas na visão, sensação de pressão em ambos os olhos, dificuldade em ler e distinguir pequenos detalhes. Após submeter-se a exames foram detectados diabetes e, concomitantemente, retinopatia diabética não proliferativa. De acordo com os dados do exame oftalmológico da cliente, sua acuidade visual no melhor olho era de 0,25, caracterizando-a como deficiente visual. Nesse momento, concluiu-se a primeira fase do processo de enfermagem, que corresponde ao exame, recolhimento de dados biográficos e de saúde do doente. Também faz parte dessa primeira fase a obtenção de informações sobre as atividades de vida da adolescente, descritas no Quadro 1.

Faz-se, no entanto, uma ressalva: as atividades de vida denominadas respiração, eliminação, higiene pessoal e vestuário, controle da temperatura do corpo, sono e morte não serão expostas no mencionado quadro, pois não foram relatados pela adolescente problemas relacionados a essas atividades de vida. Constatamos, por meio de exame físico (respiração, temperatura) e da observação e entrevista, que tais atividades de vida não sofreram alterações. A cliente não apresentou queixas relacionadas aos aspectos supracitados. Dessa forma, focamos nossas orientações nas atividades de vida que apresentaram necessidades de cuidados de enfermagem.

A análise dos dados da segunda e terceira fases, integrantes do planejamento de enfermagem, prosseguiu de forma separada, e estas foram descritas de acordo com o Modelo de Enfermagem Baseado nas Atividades da Vida Diária determinado para cada atividade. Salienta-se, porém, que as atividades de vida, assim como os outros fatores analisados, estão intimamente relacionadas. Essa forma de análise foi feita com vistas a se conseguir melhor compreensão e visualização dos resultados obtidos. 
Quadro 1 - Planejamento de enfermagem para adolescente deficiente visual e diabética de acordo com o Modelo de Enfermagem Baseado nas Atividades da Vida Diária. Fortaleza, 2006.

\begin{tabular}{|c|c|c|c|c|}
\hline Atividade de vida & $\begin{array}{c}\text { Grau de } \\
\text { dependência }\end{array}$ & Problemas & Objetivos & $\begin{array}{c}\text { Intervenções de } \\
\text { Enfermagem }\end{array}$ \\
\hline $\begin{array}{l}\text { Manut enção de } \\
\text { ambiente seguro } \\
\text { e mobilidade }\end{array}$ & $\begin{array}{l}\text { Restrições } \\
\text { deco rentes da } \\
\text { deficiência visual }\end{array}$ & $\begin{array}{l}\text { Ambiente escuro e } \\
\text { com objetos; } \\
\text { mobilidade } \\
\text { prejudicada }\end{array}$ & $\begin{array}{l}\text { Manter iluminação; } \\
\text { evitar mudanças nos } \\
\text { ambientes }\end{array}$ & $\begin{array}{l}\text { Usar lâmpada } \\
\text { fluorescente; } \\
\text { auxiliar em horários } \\
\text { notumos; orientar sobre } \\
\text { a disposição dos } \\
\text { componentes do } \\
\text { ambiente, simulando } \\
\text { treinamento para } \\
\text { independência } \\
\text { (memorização espacial) }\end{array}$ \\
\hline Alimentação & $\begin{array}{l}\text { Mãe que compra } \\
\text { e prepara os } \\
\text { alimentos }\end{array}$ & Nutrição alterada & $\begin{array}{l}\text { Inge rir alimentos } \\
\text { saudáveis; } \\
\text { controlar peso, } \\
\text { glic emia e PA }\end{array}$ & $\begin{array}{l}\text { Orientar alimentação; } \\
\text { demonstrar uso do } \\
\text { glicosímetro e aplicação } \\
\text { de insulina com o devido } \\
\text { treinamento para o } \\
\text { autocuidado }\end{array}$ \\
\hline Comunicação & $\begin{array}{l}\text { Dificuldade para } \\
\text { verbalizar } \\
\text { sentimentos }\end{array}$ & $\begin{array}{l}\text { Comunicação } \\
\text { prejudicada } \\
\text { relacionada à baixa } \\
\text { autoestima. }\end{array}$ & $\begin{array}{l}\text { Descrever sua } \\
\text { situação clínica } \\
\text { atual sem sinais de } \\
\text { ansiedade }\end{array}$ & $\begin{array}{l}\text { Estimular comunicação } \\
\text { dos sentimentos; } \\
\text { orientar sobre saúde, } \\
\text { tratamento, inclusão } \\
\text { social e autocuidado }\end{array}$ \\
\hline Trabalho e lazer & $\begin{array}{l}\text { Restrições em } \\
\text { virtude da } \\
\text { deficiência visual }\end{array}$ & $\begin{array}{l}\text { Intolerância a } \\
\text { atividade decorrente } \\
\text { da falta de políticas } \\
\text { inclusivas da pessoa } \\
\text { com deficiência visual }\end{array}$ & $\begin{array}{l}\text { Habilitar para o } \\
\text { trabalho }\end{array}$ & $\begin{array}{l}\text { Encorajar prática de } \\
\text { atividade voluntária na } \\
\text { comunidade, como } \\
\text { forma de inclusão social. }\end{array}$ \\
\hline $\begin{array}{l}\text { Expressão da } \\
\text { sexualidade }\end{array}$ & $\begin{array}{l}\text { Falta diálogo e } \\
\text { mãe superprotege }\end{array}$ & $\begin{array}{l}\text { Sexual idade alterada; } \\
\text { déficit de } \\
\text { conhecimentos sobre } \\
\text { saúde e copo }\end{array}$ & $\begin{array}{l}\text { Dialogar sobre } \\
\text { sexualidade com sua } \\
\text { mãe; } \\
\text { info mar sobre } \\
\text { sexualidade e } \\
\text { reprodução } \\
\text { humana. } \\
\end{array}$ & $\begin{array}{l}\text { Responder sobre } \\
\text { sexualidade; } \\
\text { estimular visita ao } \\
\text { ginecologista; } \\
\text { explicar prevenção de } \\
\text { câncer e DST/AIDS }\end{array}$ \\
\hline
\end{tabular}

Foram feitas quatro visitas domiciliares, e observou-se, desde a primeira, um clima de empatia estabelecido em momento anterior, sobretudo porque a adolescente já havia participado de outras atividades desempenhadas pela enfermeira. Em consideração às intervenções de enfermagem, estas visitas foram importantes para o alcance dos objetivos, pois permitiram de maneira geral, a mudança de comportamentos. Desta mudança sobressai, como consequência, a superação de alguns problemas. Isso pode ser demonstrado pelas seguintes constatações:

1. A adolescente relatou sentir mais segurança em relação ao seu ambiente doméstico, sobretudo por dois motivos: foi trocada a lâmpada fluorescente próxima à escada, melhorando a iluminação e sua visualização, e os móveis e objetos passaram a ocupar seus devidos locais e, desse modo, propiciaram a memorização espacial.

Por meio da visão, o ser humano pode identificar objetos, além de distinguir cores, formas, tamanhos e distâncias. A distância faz parte de um dos sistemas de coordenadas na relação do nosso corpo com outros corpos e objetos. Nesse processo, a visão se apresenta como um sentido de grande importância na captação de estímulos e projeções espaciais, e, desse modo, facilita o relacionamento do homem na sociedade. Enquanto a percepção de um deficiente visual atinge um raio de seis a trinta metros, a das pessoas providas de visão normal poderia atingir as estrelas. ${ }^{9}$

Em virtude das suas limitações, é preciso desenvolver a orientação e mobilidade do deficiente visual. Isto significa darIhe condições de usufruir e exercer o direito de ir e vir de forma independente. Por orientação, entende-se um recurso usado pelo deficiente visual por meio de outros sentidos para o estabelecimento das suas posições em relação com todos os objetos significativos do seu meio circundante. Quanto à mobilidade, é a capacidade de deslocamento do ponto onde se encontra o indivíduo para alcançar outra zona do meio circundante. ${ }^{10}$

Toda pessoa tem direito à liberdade de ir e vir. Esse direito é assegurado legalmente. No Brasil, a Constituição protege o cidadão do cerceamento da liberdade de locomoção, concedendo-the habeas corpus em caso de impedimento. Logo, criar barreiras que limitem ou impeçam a orientação e mobilidade de uma pessoa deficiente visual é desumano e inconstitucional. 
2. No concernente à comunicação prejudicada relacionada à alteração da autoestima, percebe-se, também, um bom nível de evolução, em decorrência de melhor expressão de sentimentos e aquisição de conhecimentos sobre sua doença e prognóstico. Ademais, observou-se comportamento de busca de saúde.

Conforme se sabe, a expressão verbal é uma das características mais fascinantes do ser humano. Contudo, a linguagem não verbal é um elemento fundamental na comunicação com os outros. Entre as comunicações não verbais, incluem-se sinais que produzimos, gestos e imagens que criamos ou percebemos. Elas acontecem por meio das mãos, da cabeça, do rosto, da boca, enfim, ocorrem pela expressão do corpo. Apesar de nem sempre a expressão não verbal possuir a objetividade das palavras, é carregada de significados. ${ }^{11}$

0 olhar, por exemplo, constitui um elemento único e primário das relações interpessoais. São numerosas as funções da interação visual: o olhar desempenha um papel decisivo na comunicação de atitudes interpessoais e no estabelecimento de relações; está, além disso, estreitamente ligado à comunicação verbal, no decurso da qual é utilizado para obter informações retroativas sobre as reações do interlocutor enquanto se vai falando para obter novos elementos de informação acerca daquilo que é dito. 0 olhar é, enfim, usado como sinal para preparar encontros, para saudar e para indicar se a ideia expressa foi compreendida pelo outro. ${ }^{9}$ Estas são funções diferentes, e é por isso que o estudo do olhar se revela notavelmente complexo, dada a dificuldade de distinguir a função específica por ele ocupada em cada momento particular.

Na interação com a deficiente visual, manteve-se a atenção para o que ela expressava verbalmente ou não, pois este tipo de deficiência impossibilita o não vidente de perceber a gestualidade do interlocutor. Além disso, os gestos e maneirismos apresentados pelo não vidente podem não ter a mesma significação para uma pessoa vidente.

3. No relacionado à nutrição, houve a inserção de frutas no cardápio diário da adolescente, foram evitados o açúcar, as gorduras e as frituras, porém as três refeições principais continuam sendo feitas de forma não balanceada.

A doença acarreta mudanças significativas na relação do diabético com seu próprio corpo e com o mundo ao seu redor. É, sobretudo, por meio das restrições no comportamento alimentar que o diabético toma consciência das suas limitações. Por essa razão, o conflito entre o desejo alimentar e a necessidade imperiosa de contê-lo está sempre presente na vida cotidiana dele. 0 desejo alimentar faz o doente sofrer, reprimir, salivar, esquecer, transgredir, mentir, negar, admitir, sentir prazer, se controlar e sentir culpa. Ao mesmo tempo, esse desejo o faz feliz de uma forma que só ele sabe descrever. ${ }^{12}$

Em uma das falas da adolescente, percebeu-se uma sensação de frustração, pois ela se ressente de não poder comer determinados alimentos da sua preferência e nos momentos desejados. Como podemos observar a seguir:
Eu fico triste porque minha mãe não me deixa comer doces, sorvetes, chicletes, refrigerante, e todo mundo da minha escola come isso. Na hora do recreio eu sinto vontade de comer coisas na cantina, mas não posso. Só como o que levo de casa. (F.L.S)

Nesse contexto, a doença surge como uma ameaça à autonomia individual, além de representar um fator limitante para a sua qualidade de vida. Nessa perspectiva, os maiores problemas explicitados são a reorganização do cardápio e a busca de apoio profissional para ajudá-la na resolução nas dificuldades apresentadas.

4. Quanto ao uso do seu glicosímetro, a doente demonstrou usá-lo de forma correta, assim como aplicou a insulina e relatou os locais de aplicação/rodízio e valores glicêmicos e de pressão arterial normais. Após a execução das técnicas, pudemos concluir que as orientaç̃̃es repassadas durante as visitas domiciliares foram eficazes, tornando a cliente independente em relação a este aspecto de autocuidado.

Com a proposta de intensificação de um controle rigoroso da doença obtida por meio da insulinoterapia, foi recomendado pelo Diabetes Control and Complication Trial (DDCT) aumentar as aplicações diárias de insulina de uma para três vezes. ${ }^{13}$ Portanto, o controle metabólico rigoroso implica a manutenção da glicemia o mais próximo do normal a maior parte do tempo, com a normalização da glico-hemoglobina. Nesse aspecto, ressalta-se uma dificuldade, qual seja, o custo elevado dos materiais necessários para manter 0 diabetes, como fitas, glicosímetro, lancetas, insulina, seringas, somado ao fornecimento irregular desses materiais pelo Sistema Único de Saúde, compromete a prática de insulinoterapia intensiva ${ }^{14}$.

5. No relacionado à manutenção de hábitos saudáveis, a adolescente relatou sentir-se mais disposta, pois está caminhando assiduamente (três vezes na semana, durante trinta minutos). Sua mãe comprou roupas de ginástica novas, e isto é mais um incentivo à realização deste saudável hábito.

A atividade física exerce efeito oposto ao do sedentarismo. Aumenta o gasto calórico e melhora o transporte e a captação de insulina. Tanto os exercícios aeróbicos quanto os resistidos promovem um aumento do metabolismo basal conhecido como metabolismo de repouso, o qual é responsável por $60 \%$ a $70 \%$ do gasto energético total, contribuindo para a perda de peso e diminuição do risco de desenvolver diabetes, hipertensão e outras doenças. ${ }^{15}$

Conforme divulgado, a adoção de um estilo de vida não sedentário, calcado na prática regular de atividade física, elimina a possibilidade de desenvolvimento da maior parte das doenças crônicas degenerativas, além de servir como elemento promotor de mudanças em relação a fatores de risco para inúmeras outras doenças. Sugere-se, inclusive, que a prática regular de atividade física seja adotada como forma de prevenção das doenças crônicas degenerativas, o equivalente ao que a imunização representa na tentativa de controle das doenças infectocontagiosas. ${ }^{16}$ 
6. Como recomendado, a adolescente procurou um serviço de ginecologia e marcou data para a realização do exame de prevenção de câncer de colo uterino. Vale salientar que ela fará este exame pela primeira vez. Tal atitude resulta também do diálogo sobre as questões de sexualidade entre ela e a genitora, a qual a acompanha em suas atividades.

\section{CONSIDERAÇÕES FINAIS}

Segundo evidenciado, o uso do Modelo de Enfermagem Baseado nas Atividades da Vida Diária é um instrumento válido para acompanhamento da adolescente acometida por doença, pois permitiu e promoveu o cuidado e comunicação objetiva entre pesquisador e adolescente deficiente visual. Ademais, 0 processo de enfermagem baseado no Modelo forneceu subsídios para a aplicação sistemática da assistência de enfermagem prestada à adolescente, proporcionando mudanças necessárias na fase de planejamento de cuidados a ela dispensados. A

\section{REFERÊNCIAS}

1. Ministério da Saúde (BR). Secretaria de Atenção à Saúde do Adolescente e do Jovem. Marco legal: saúde, um direito de adolescentes. Brasília (DF); 2005. [citado 20 jul 2008]. Disponível em: http://dtr2001.saude.gov.br/editora/produtos/ livros/pdf/05_0011_M.pdf

2. Ministério da Saúde (BR). Marco teórico e referencial: saúde sexual e saúde reprodutiva de adolescentes e jovens. Brasília (DF); 2006. [citado 20 jul 2008]. Disponível em: http:// dtr2001.saude.gov.br/editora/produtos/livros/pdf/ 06_0611_M.pdf

3. Ministério da Saúde (BR). Saúde integral de adolescentes e jovens: orientações para a organização de serviços de saúde. Brasília (DF); 2005. [citado 20 jul 2008]. Disponível em: http:/ /portal.saude.gov.br/portal/arquivos/pdf/saude_integral.pdf

4. Roper N, Logan W, Tierney AJ. 0 modelo de enfermagem: baseado nas atividades de vida diária. Lisboa: Climepsi; 2001.

5. Smeltzer SC, Bare BG. Tratado de enfermagem médicocirúrgica. 10ª ed. Rio de Janeiro (RJ): Guanabara Koogan; 2006.

6. Vilarinho RMF, Lisboa MTL. Assistência de enfermagem na prevenção do diabetes mellitus tipo 2: uma questão da atualidade. Esc Anna Nery Rev Enferm 2008 jun; 9 (1): 10307.

7. Lobiondo-Wood G, Haber J. Pesquisa em enfermagem: métodos, avaliação crítica e utilização. $4^{\mathrm{a}}$ ed. Rio de Janeiro (RJ): Guanabara Koogan; 2001.

8. Ministério da Saúde (BR). Conselho Nacional de Saúde. Comissão Nacional de Ética em Pesquisa. Resolução n 196 , de 10 de outubro de 1996: aprova as diretrizes e normas regulamentadoras de pesquisa envolvendo seres humanos. Brasília (DF); 1996.

9. Hall ET. A dimensão oculta. Lisboa: Relógio D’Água; 1986.

10. Fonseca V. Psicomotricidade. São Paulo (SP): Martins Fontes; 1988. adolescente demonstrou boa apreensão no referente às intervenções estabelecidas e mostrou-se mais segura e autoconfiante. Isto repercute em aumento da autoestima e desempenho de atividades de forma mais independente. Portanto, é um demonstrativo da efetividade do modelo adotado.

Este artigo representa um estudo preliminar no levantamento de possíveis problemas de enfermagem identificados em uma adolescente deficiente visual com diabetes mellitus. Consoante se entendeu, a identificação desses problemas não pode constituir uma fase isolada de todo 0 processo assistencial; ela deve ser utilizada com vistas a direcionar a ação de enfermagem. Cabe ressaltar, ainda, a necessidade de outros estudos nessa área junto a adolescentes deficientes visuais e diabéticas para subsidiar a assistência de enfermagem sistematizada e baseada em fundamentos científicos.

11.Almeida CB. Características da comunicação não-verbal entre o enfermeiro e o cego [dissertação de mestrado]. Fortaleza (CE): Faculdade de Enfermagem/UFC; 2005.

12.Santos ECB, Zanetti ML, Otero LM, Santos MA. 0 cuidado sob a ótica do paciente diabético e de seu principal cuidador. Rev Latino-am Enfermagem. 2005 maio/jun; 13 (3): 397-406.

13. American Diabetes Association. Implementation of treatment protocols in the diabetes control and complications trial. Diabetes Care [on-line] 2003; [citado 20 jul 2008]; 26(supp1): [aprox.3 telas]. Disponível em: http://care.diabetesjournals.org/content/26/suppl_1/ s25.full.pdf+html

14.Castro ARV, Graziano KU, Grossi SA. Alterações nos locais de aplicação de insulina e nas seringas reutilizadas pelos pacientes diabéticos. Rev Gaucha Enferm 2006 mar; 27(1): 27-34.

15.Ciolac EG, Guimarães GV. Exercício físico e síndrome metabólica. Rev Bras Med Esporte. [on-line] 2004 jul/ago; [citado 20 jul 2008]; 10(4): [aprox.6 telas]. Disponível em: http://www.scielo.br/pdf/rbme/ v10n4/22048.pdf

16. Choayeb N, Barros Neto TL. 0 exercício - preparação fisiológica, avaliação médica, aspectos especiais e preventivos. São Paulo (SP): Atheneu; 1999.

\section{NOTA}

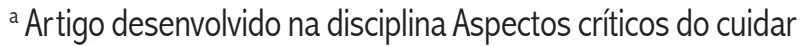
em enfermagem do Programa de Pós-Graduação em Enfermagem da Universidade Federal do Ceará (UFC). 